\title{
Ergodic Hypothesis and Equilibrium Statistical Mechanics in the Quantum Mechanical World View
}

\author{
Shiro Ishikawa \\ Department of Mathematics, Faculty of Science and Technology, Keio University, Yokohama, Japan \\ Email: ishikawa@math.keio.ac.jp
}

Received December 20, 2011; revised January 25, 2012; accepted February 28, 2012

\begin{abstract}
In this paper, we study and answer the following fundamental problems concerning classical equilibrium statistical mechanics: 1): Is the principle of equal a priori probabilities indispensable for equilibrium statistical mechanics? 2): Is the ergodic hypothesis related to equilibrium statistical mechanics? Note that these problems are not yet answered, since there are several opinions for the formulation of equilibrium statistical mechanics. In order to answer the above questions, we first introduce measurement theory (i.e., the theory of quantum mechanical world view), which is characterized as the linguistic turn of quantum mechanics. And we propose the measurement theoretical foundation of equilibrium statistical mechanics, and further, answer the above 1) and 2), that is, 1) is "No", but, 2) is "Yes".
\end{abstract}

Keywords: The Copenhagen Interpretation; Probability; Operator Algebra; Ergodic Theorem; Quantum and Classical Measurement Theory; Liouville's Theorem; The Law of Increasing Entropy

\section{Introduction}

Recently in [1-6] we proposed (classical and quantum) measurement theory, which is characterized as the linguistic (or, metaphysical) turn of quantum mechanics. As seen in [1-6], this theory includes several conventional system theories (e.g, quantum system theory, statistics, dynamical system theory and so on). Also, for the philosophical aspect of measurement theory (called the quantum mechanical world view), see [5]. And thus, we believe that measurement theory is one of the most fundamental theories in science.

Note that there are several opinions ( $c f .[2,3,7-9]$ ) for the formulation of equilibrium statistical mechanics, and hence, there are several opinions for the problems 1) and 2) mentioned in the abstract.

The purpose of this paper is to reinforce our method [2, $3]$, or equivalently, to clarify the principle of equal probability and the ergodic hypothesis in the light of measurement theory [4,5] (i.e., Axioms 1 and 2, Interpretation (E) mentioned in the following section).

\section{Measurement Theory (Axioms 1 and 2, Interpretation)}

In this section, according to [4], we explain the outline of measurement theory (or in short, MT).

Measurement theory is, by an analogy of quantum mechanics (or, as a linguistic turn of quantum mechanics), constructed as the mathematical theory formulated in a certain $C^{*}$-algebra $A$ (i.e., a norm closed subalgebra in the operator algebra $B(H)$ composed of all bounded operators on a Hilbert space $H, c f .[10,11])$ as follows:

$$
\text { (A) } \underset{\text { (language) }}{[\mathrm{MT}]}=\underset{(\text { Axiom 1) }}{\underset{(\text { Axiom 2) }}{\operatorname{measurement}}]}+\underset{\text { causality }}{[\text { con }}
$$

For completeness, note that measurement theory (A) is not physics but a kind of language based on "the quantum mechanical world view" (cf. [5]).

When $A=B_{c}(H)$, the $C^{*}$-algebra composed of all compact operators on a Hilbert space $H$, the (A) is called quantum measurement theory (or, quantum system theory), which can be regarded as the linguistic aspect of quantum mechanics. Also, when $A$ is commutative (that is, when $A$ is characterized by $C_{0}(\Omega)$, the $C^{*}$-algebra composed of all continuous complex-valued functions vanishing at infinity on a locally compact Hausdorff space $\Omega$ (cf. [10])), the (A) is called classical measurement theory. Thus, we have the following classification:

$\left(\mathrm{B}_{1}\right) \mathrm{MT}\left\{\begin{array}{l}\text { quantum MT } \quad\left(\text { when } A=B_{C}(H)\right) \\ \text { classical MT }\left(\text { when } A=C_{0}(\Omega)\right)\end{array}\right.$

Hence, we consider that

$\left(B_{2}\right)$ the theory of classical mechanical world view $=$ classical measurement theory in $\left(B_{1}\right)$ $\subset$ MT (i.e., the theory of quantum mechanical world view).

And we never consider that

$\left(\mathrm{B}_{3}\right)$ the theory of classical mechanical world view 
$=$ Something like Newtonian mechanics

+ Kolmogorov's probability theory [12],

which may be usually called dynamical system theory. It should be noted that Ruelle's method (cf. [7]), which is the most authorized approach to equilibrium statistical mechanics, is based on the $\left(\mathrm{B}_{3}\right)$. Thus, our interest in this paper may be regarded as our method $[2,3]$ in $\left(B_{2}\right)$ versus Ruelle's method [7] in $\left(\mathrm{B}_{3}\right)$.

Now we shall explain measurement theory (A). Let $A(\subseteq B(H))$ be a $C^{*}$-algebra, and let $A^{*}$ be the dual Banach space of $A$. That is, $A^{*}=\{\rho \mid \rho$ is a continuous linear functional on $A\}$, and the norm $\|\rho\|_{A^{*}}$ is defined by $\sup \left\{|\rho(F)|: F \in A\right.$ such that $\left.\|F\|_{A}\left(=\|F\|_{B(H)}\right) \leq 1\right\}$.

Define the mixed state $\rho\left(\in A^{*}\right)$ such that $\|\rho\|_{A^{*}}=1$ and $\rho(F) \geq 0$ for all $F \in A$ such that $F \geq 0$. And put $\mathfrak{S}^{m}\left(A^{*}\right)=\left\{\rho \in A^{*} \mid \rho\right.$ is amixed state $\}$.

For example, note that $\mathfrak{S}^{m}\left(C_{0}(\Omega)^{*}\right)=M_{+1}^{m}(\Omega)=$ $\{\rho \mid \rho$ is a measure on $\Omega$ such that $\rho(\Omega)=1\}$. A mixed state $\rho\left(\in \mathfrak{S}^{m}\left(A^{*}\right)\right)$ is called a pure state if it satisfies that $\rho=\theta \rho_{1}+(1-\theta) \rho_{2}$ for some

$\rho_{1}, \rho_{2} \in \mathfrak{S}^{m}\left(A^{*}\right)$ and $0<\theta<1$ implies $\rho=\rho_{1}=\rho_{2}$. Put $\mathfrak{S}^{p}\left(A^{*}\right)=\left\{\rho \in \mathfrak{S}^{m}\left(A^{*}\right) \mid \rho\right.$ is a purestate $\}$,

which is called a state space. It is well known (cf. [10]) that $\mathfrak{S}^{p}\left(B_{c}(H)^{*}\right)=\{|u\rangle\langle u|$ (i.e., the Dirac notation) $\left.\mid\|u\|_{H}=1\right\}$, and $\mathfrak{S}^{p}\left(C_{0}(\Omega)^{*}\right)=\left\{\delta_{\omega_{0}} \mid \delta_{\omega_{0}}\right.$ is a point measure at $\omega_{0} \in \Omega$, where $\int_{\Omega} f(\omega) \delta_{\omega_{0}}(d \omega)=f\left(\omega_{0}\right)$ $\left(\forall f \in C_{0}(\Omega)\right)$. The latter implies that $\mathfrak{S}^{p}\left(C_{0}(\Omega)^{*}\right)$ can be also identified with $\Omega$ (called a spectrum space or maximal ideal space) such as

$$
\underset{\text { (state space) }}{\mathfrak{S}^{p}}\left(C_{0}(\Omega)^{*}\right) \ni \delta_{\omega} \leftrightarrow \omega \in \underset{\text { (spectrum space) }}{\Omega}
$$

In this sense, the $\Omega$ is also called a state space in classical measurement theory.

Here, assume that the $C^{*}$-algebra $A(\subseteq B(H))$ has the identity $I$. This assumption is not unnatural, since, if $I \notin A$, it suffices to reconstruct the above $A$ such that it includes $A \bigcup\{I\}$. According to the noted idea (cf. [13]) in quantum mechanics, an observable $\mathrm{O}:=(X, F, F)$ in $A$ is defined as follows:

$\left(\mathrm{C}_{1}\right)$ [Field] $X$ is a set, $F\left(\subseteq 2^{X}\right.$, the power set of $X$ ) is a field of $X$, that is, " $\Xi_{1}, \Xi_{2} \in F \Rightarrow \Xi_{1} \cup \Xi_{2} \in F$ ", “ $\Xi \in F \Rightarrow X \backslash \Xi \in F$ ".

$\left(\mathrm{C}_{2}\right)$ [Finite additivity] $F$ is a mapping from $F$ to $A$ satisfying: 1): for every $\Xi \in F, F(\Xi)$ is a non-negative element in $A$ such that $0 \leq F(\Xi) \leq I, 2): F(\varnothing)=0$ and $F(X)=I$, where 0 and $I$ is the 0 -element and the identity in $A$ respectively. 3): for any $\Xi_{1}, \Xi_{2} \in F$ such that $\Xi_{1} \cap \Xi_{2}=\varnothing$, it holds that $F\left(\Xi_{1} \cup \Xi_{2}\right)=F\left(\Xi_{1}\right)+F\left(\Xi_{2}\right)$.

For the further argument (e.g., $\sigma$-field, countably additivity, the $W^{*}$-algebraic formulation, etc.), see [4, $6]$.

With any system $S$, a $C^{*}$-algebra $A(\subseteq B(H))$ can be associated in which the measurement theory (A) of that system can be formulated. A state of the system $S$ is represented by an element $\rho\left(\in \mathfrak{S}^{p}\left(A^{*}\right)\right)$ and an observable is represented by an observable $\mathrm{O}:=(X, F, F)$ in $A$. Also, the measurement of the observable $\mathrm{O}$ for the system $S$ with the state $\rho$ is denoted by $\mathrm{M}_{A}\left(\mathrm{O}, S_{[\rho]}\right)$ (or more precisely, $\left.\mathrm{M}_{A}\left(\mathrm{O}:=(X, F, F), S_{[\rho]}\right)\right)$. An observer can obtain a measured value $x(\in X)$ by the measurement $\mathrm{M}_{A}\left(\mathrm{O}, S_{[\rho]}\right)$.

The Axiom 1 presented below is a kind of mathematical generalization of Born's probabilistic interpretation of quantum mechanics. And thus, it is a statement without reality.

Axiom 1 [Measurement]. The probability that a measured value $x(\in X)$ obtained by the measurement $\mathrm{M}_{A}\left(\mathrm{O}:=(X, F, F), S_{[\rho 0]}\right)$ belongs to a set $\Xi(\in F)$ is given by $\rho_{0}(F(\Xi))$.

Next, we explain Axiom 2 in (A). Let $(T, \leq)$ be a tree, i.e., a partial ordered set such that $t_{1} \leq t_{3}$ and $t_{2} \leq t_{3}$ implies $t_{1} \leq t_{2}$ or $t_{2} \leq t_{1}$. Assume that there exists an element $t_{0} \in T$, called the root of $T$, such that $t_{0} \leq t$ $(\forall t \in T)$ holds. Put $T_{\leq}^{2}=\left\{\left(t_{1}, t_{2}\right) \in T^{2} \mid t_{1} \leq t_{2}\right\}$. The family $\left\{\Phi_{t_{1}, t_{2}}: A_{t_{2}} \rightarrow A_{t_{1}}\right\}_{\left(t_{1}, t_{2}\right) \in T_{\leq}^{2}}$ is called a Markov relation ( due to the Heisenberg picture), if it satisfies the following conditions $\left(\mathrm{D}_{1}\right)$ and $\left(\mathrm{D}_{2}\right)$.

$\left(D_{1}\right)$ With each $t \in T$, a $C^{*}$-algebra $A_{t}$ is associated.

$\left(\mathrm{D}_{2}\right)$ For every $\left(t_{1}, t_{2}\right) \in T_{\leq}^{2}$, a Markov operator $\Phi_{t_{1}, t_{2}}: A_{t_{2}} \rightarrow A_{t_{1}}$ is defined. And it satisfies that $\Phi_{t_{1}, t_{2}} \Phi_{t_{2}, t_{3}}=\Phi_{t_{1}, t_{3}}$ holds for any $\left(t_{1}, t_{2}\right),\left(t_{2}, t_{3}\right) \in T_{\leq}^{2}$.

The family of dual operators $\left\{\Phi_{t_{1}, t_{2}}^{*}: \mathfrak{S}^{m}\left(A_{t_{1}}^{*}\right) \rightarrow \mathfrak{S}^{m}\left(A_{t_{2}}^{*}\right)\right\}_{\left(t_{1}, t_{2}\right) \in T_{\leq}^{2}}$ is called a dual Markov relation (due to the Schrödinger picture). Also, when $\Phi_{t_{1}, t_{2}}^{*}\left(\mathfrak{S}^{p}\left(A_{t_{1}}^{*}\right)\right) \subseteq\left(\mathfrak{S}^{p}\left(A_{t_{2}}^{*}\right)\right)$ holds for any $\left(t_{1}, t_{2}\right) \in T_{\leq}^{2}$, the Markov relation is said to be deterministic.

Now Axiom 2 in the measurement theory (A) is presented as follows:

Axiom 2 [Causality]. The causality is represented by a Markov relation $\left\{\Phi_{t_{1}, t_{2}}: A_{t_{2}} \rightarrow A_{t_{1}}\right\}_{\left(t_{1}, t_{2}\right) \in T_{\leq}^{2}}$.

Further, we have to explain how to use Axioms 1 and 2 as follows. That is, we present the following interpretation $(E)\left[=\left(E_{1}\right)-\left(E_{4}\right)\right]$, which is characterized as a kind of linguistic turn of so-called Copenhagen interpretation. That is, we propose $(c f .[4,5])$ :

$\left(E_{1}\right)$ Consider the dualism composed of observer and system(=measuring object). And therefore, observer and 
system must be absolutely separated.

$\left(E_{2}\right)$ Only one measurement is permitted. And thus, the state after a measurement is meaningless since it can not be measured any longer. Also, the causality should be assumed only in the side of system, however, a state never moves. Thus, the Heisenberg picture should be adopted.

$\left(E_{3}\right)$ Also, the observer does not have the space-time. Thus, the question: When and where is a measured value obtained? is out of measurement theory.

Thus, we say that

$\left(E_{4}\right)$ there is no probability without measurement.

Since measurement theory is a kind of language, the spirit is based on Wittgenstein's famous statement: "the limits of my language mean the limits of my world". Thus, the $\left(E_{3}\right)$ says, for example, that Schrödinger's cat is out of the world of measurement theory.

\section{Equilibrium Statistical Mechanics in Measurement Theory}

\subsection{Statements Concerning Axiom 2 (Dynamical Aspect; Ergodic Hypothesis)}

3.1.1. Equilibrium Statistical Mechanical Phenomena Assume that about $N\left(\approx 10^{24}\right)$ particles (for example, hydrogen molecules) move in a box. It is natural to assume the following phenomenas 1)-4)

1) Every particle obeys Newtonian mechanics.

2) Every particle moves uniformly in the box. For example, a particle does not halt in a corner.

3) Every particle moves with the same statistical behavior concerning time.

4) The motions of particles are (approximately) independent of each other.

In what follows we shall devote ourselves to the problem:

(F) how to describe the above equilibrium statistical mechanical phenomenas 1)-4) in terms of measurement theory.

For completeness, again note that measurement theory is a kind of language.

\subsubsection{About 1)}

In Newtonian mechanics, any state of a system composed of $N\left(\approx 10^{24}\right)$ particles is represented by a point $(q, p)$ $\left(\equiv(\right.$ position, momentum $\left.)=\left(q_{1 n}, q_{2 n}, q_{3 n}, p_{1 n}, p_{2 n}, p_{3 n}\right)_{n=1}^{N}\right)$ in a phase (or state) space $\mathbb{R}^{6 N}$. Let $H: \mathbb{R}^{6 N} \rightarrow \mathbb{R}$ be a Hamiltonian such that

$$
\begin{aligned}
& H\left(\left(q_{1 n}, q_{2 n}, q_{3 n}, p_{1 n}, p_{2 n}, p_{3 n}\right)_{n=1}^{N}\right) \\
& =\left[\sum_{n=1}^{N} \sum_{k=1,2,3} \frac{\left(p_{k n}\right)^{2}}{2 \times \text { particle'mass }}\right]+U\left(\left(q_{1 n}, q_{2 n}, q_{3 n}\right)_{n=1}^{N}\right) .
\end{aligned}
$$

Fix $E>0$. And define the measure $v_{E}$ on the energy surface $\Omega_{E}\left(\equiv\left\{(q, p) \in \mathbb{R}^{6 N} \mid H(q, p)=E\right\}\right)$ such that

$$
\begin{aligned}
& v_{E}(B)=\int_{B}|\nabla H(q, p)|^{-1} \mathrm{~d} m_{6 N-1} \\
& \left(\forall B \in B_{\Omega_{E}}, \text { the Borel field of } \Omega_{E}\right)
\end{aligned}
$$

where

$$
|\nabla H(q, p)|=\left[\sum_{n=1}^{N} \sum_{k=1,2,3}\left\{\left(\frac{\partial H}{\partial p_{k n}}\right)^{2}+\left(\frac{\partial H}{\partial q_{k n}}\right)^{2}\right\}\right]^{1 / 2}
$$

and $d m_{6 N-1}$ is the usual surface measure on $\Omega_{E}$. Let $\left\{\psi_{t}^{E}\right\}_{-\infty<t<\infty}$ be the flow on the energy surface $\Omega_{E}$ induced by the Newton equation with the Hamiltonian $H$, or equivalently, Hamilton's equation:

$$
\begin{gathered}
\frac{\mathrm{d} q_{k n}}{\mathrm{~d} t}=\frac{\partial H}{\partial p_{k n}}, \quad \frac{\mathrm{d} p_{k n}}{\mathrm{~d} t}=-\frac{\partial H}{\partial q_{k n}}, \\
(k=1,2,3, n=1,2, \cdots, N) .
\end{gathered}
$$

Liouville's theorem ( $c f .[9])$ says that the measure $v_{E}$ is invariant concerning the flow $\left\{\psi_{t}^{E}\right\}_{-\infty<t<\infty}$. Defining the normalized measure $\bar{v}_{E}$ such that $\bar{v}_{E}=\frac{v_{E}}{v_{E}\left(\Omega_{E}\right)}$, we have the normalized measure space $\left(\Omega_{E}, B_{\Omega_{E}}, \bar{v}_{E}\right)$.

Putting $A=C_{0}\left(\Omega_{E}\right)=C\left(\Omega_{E}\right)$ (from the compactness of $\left.\Omega_{E}\right), T=\mathbb{R}, \omega_{t}=(q(t), p(t)), \phi_{t_{1}, t_{2}}=\psi_{t_{2}-t_{1}}^{E}$, $\Phi_{t_{1}, t_{2}}^{*} \delta_{\omega_{t_{1}}}=\delta_{\phi_{t_{1}, t_{2}}\left(\omega_{t_{1}}\right)} \quad\left(\forall \omega_{t_{1}} \in \Omega_{E}\right)$, we define the deterministic Markov relation $\left\{\Phi_{t_{1}, t_{2}}: C\left(\Omega_{E}\right) \rightarrow C\left(\Omega_{E}\right)\right\}_{\left(t_{1}, t_{2}\right) \in T_{\leq}^{2}}$ in Axiom 2.

\subsubsection{About 2)}

Now let us begin with the well-known ergodic theorem (cf. [9, 14]).

For example, consider one particle $\mathrm{P}_{1}$. Put $S_{R_{1}}=\left\{\omega \in \Omega_{E} \mid \quad\right.$ a state $\omega$ such that the particle $P_{1}$ always stays a corner of the box $\}$. Clearly, it holds that $S_{P_{1}} \subsetneq \Omega_{E}$. Also, if $\psi_{t}^{E}\left(S_{P_{1}}\right) \subseteq S_{P_{1}} \quad(0 \leqq \forall t<\infty)$, then the particle $P_{1}$ must always stay a corner. This contradicts 2). Therefore, 2) means the following:

2)' [Ergodic property]: If a compact set $S\left(\subseteq \Omega_{E}, S \neq \varnothing\right)$ satisfies $\psi_{t}^{E}(S) \subseteq S \quad(0 \leqq \forall t<\infty)$, then it holds that $S=\Omega_{E}$.

The ergodic theorem (cf. [14]) says that the above 2)' is equivalent to the following equality:

$$
\begin{gathered}
\int_{\substack{\Omega_{E} \\
\text { (state) space average) }}} f(\omega) \bar{v}_{E}(\mathrm{~d} \omega)=\lim _{T \rightarrow \infty} \frac{1}{T} \int_{\alpha}^{\alpha+T} f\left(\psi_{t}^{E}\left(\omega_{0}\right)\right) \mathrm{d} t \\
\left(\forall \alpha \in \mathbb{R}, \forall f \in C\left(\Omega_{E}\right), \quad \forall \omega_{0} \in \Omega_{E}\right)
\end{gathered}
$$

After all, the ergodic property says that if $T$ is su- 
fficiently large, it holds that

$$
\int_{\Omega_{E}} f(\omega) \bar{v}_{E}(\mathrm{~d} \omega) \approx \frac{1}{T} \int_{\alpha}^{\alpha+T} f\left(\psi_{t}^{E}\left(\omega_{0}\right)\right) \mathrm{d} t .
$$

Put $\bar{m}_{T}(\mathrm{~d} t)=\frac{\mathrm{d} t}{T}$. The probability space

$\left([\alpha, \alpha+T], B_{[\alpha, \alpha+T]}, \bar{m}_{T}\right)$ (or equivalently,

$\left.\left([0, T], B_{[0, T]}, \bar{m}_{T}\right)\right)$ is called a (normalized) first staying time space, also, the probability space $\left(\Omega_{E}, B_{\Omega_{E}}, \bar{v}_{E}\right)$ is called a (normalized) second staying time space. Note that these mathematical probability spaces are not related to probability" ( $c f$. Section 3.2).

Remark 1. [About 2)']. In [2,3], we started form the mathematical statement 2)'. In this paper, this is improved by the phenomenological statement 2).

\subsubsection{About 3) and 4)}

Put $K_{N}=\left\{1,2, \cdots, N\left(\approx 10^{24}\right)\right\}$. For each $k\left(\in K_{N}\right)$, define the coordinate map $\pi_{k}: \Omega_{E}\left(\subset \mathbb{R}^{6 N}\right) \rightarrow \mathbb{R}^{6}$ such that

$$
\begin{aligned}
& \pi_{k}(\omega)=\pi_{k}(q, p) \\
& =\pi_{k}\left(\left(q_{1 n}, q_{2 n}, q_{3 n}, p_{1 n}, p_{2 n}, p_{3 n}\right)_{n=1}^{N}\right) \\
& =\left(q_{1 k}, q_{2 k}, q_{3 k}, p_{1 k}, p_{2 k}, p_{3 k}\right)
\end{aligned}
$$

for all

$$
\begin{aligned}
& \omega=(q, p)=\left(q_{1 n}, q_{2 n}, q_{3 n}, p_{1 n}, p_{2 n}, p_{3 n}\right)_{n=1}^{N} \\
& \in \Omega_{E}\left(\subset \mathbb{R}^{6 N}\right)
\end{aligned}
$$

Also, for any subset $K\left(\subseteq K_{N}=\left\{1,2, \cdots, N\left(\approx 10^{24}\right)\right\}\right)$, define the distribution map

$D_{K}^{(\cdot)}: \Omega_{E}\left(\subset \mathbb{R}^{6 N}\right) \rightarrow M_{+1}^{m}\left(\mathbb{R}^{6}\right)$ such that

$$
D_{K}^{(q, p)}=\frac{1}{\#[K]} \sum_{k \in K} \delta_{\pi_{k}(q, p)}\left(\forall(q, p) \in \Omega_{E}\left(\subset \mathbb{R}^{6 N}\right)\right)
$$

where $\#[K]$ is the number of the elements of the set $K$. Let $\omega_{0}\left(\in \Omega_{E}\right)$ be a state. For each $n\left(\in K_{N}\right)$, we define the map $X_{n}^{\omega_{0}}:[0, T] \rightarrow \mathbb{R}^{6}$ such that

$$
X_{n}^{\omega_{0}}(t)=\pi_{n}\left(\psi_{t}^{E}\left(\omega_{0}\right)\right) \quad(\forall t \in[0, T])
$$

And, we regard $\left\{X_{n}^{\omega_{0}}\right\}_{n=1}^{N}$ as random functions on the probability space $\left([0, T], B_{[0, T]}, \bar{m}_{T}\right)$. Then, 3) and 4) respectively means

3)' $\left\{X_{n}^{\omega_{0}}\right\}_{n=1}^{N}$ is a sequence with the approximately identical distribution concerning time. In other words, there exists a normalized measure $\rho_{E}$ on $\mathbb{R}^{6}$ (i.e., $\rho_{E} \in M_{+1}^{m}\left(\mathbb{R}^{6}\right)$ ) such that:

$$
\begin{aligned}
& \bar{m}_{T}\left(\left\{t \in[0, T]: X_{n}^{\omega_{0}}(t) \in \Xi\right\}\right) \approx \rho_{E}(\Xi) \\
& \left(\forall \Xi \in B_{\mathbb{R}^{6}}, n=1,2, \cdots, N\right)
\end{aligned}
$$

4)' $\left\{X_{n}^{\omega_{0}}\right\}_{n=1}^{N}$ is approximately independent, in the sense that, for any $K_{0} \subset\left\{1,2, \cdots, N\left(\approx 10^{24}\right)\right\}$ such that $1 \leqq \#\left[K_{0}\right] \ll N \quad$ (that is, $\left.\frac{\#\left[K_{0}\right]}{N} \approx 0\right)$, it holds that

$$
\begin{aligned}
& \bar{m}_{T}\left(\left\{t \in[0, T]: X_{k}^{\omega_{0}}(t) \in \Xi_{k}\left(\in B_{\mathbb{R}^{6}}\right), k \in K_{0}\right\}\right) \\
& \approx \underset{k \in K_{0}}{\times \bar{m}_{T}}\left(\left\{t \in[0, T]: X_{k}^{\omega_{0}}(t) \in \Xi_{k}\left(\in B_{\mathbb{R}^{6}}\right)\right\}\right) .
\end{aligned}
$$

The following important remark was missed in [2,3]. This is the advantage of our method in comparison with Ruelle's method ( $c f$. [7]).

Remark 2. [About the time interval $[0, T]$ ]. For example, as one of typical cases, consider the motion of $10^{24}$ particles in a cubic box (whose long side is $0.3 \mathrm{~m}$ ). It is usual to consider that averaging velocity $=5 \times 10^{2} \mathrm{~m} / \mathrm{s}$, mean free path $=10^{-7} \mathrm{~m}$. And therefore, the collisions rarely happen among $\#\left[K_{0}\right]$ particles in the time interval $[0, T]$, and therefore, the motion is "almost independent". For example, putting $\#\left[K_{0}\right]=10^{10}$, we can cal-culate the number of times a certain particle collides with $K_{0}$-particles in $[0, T]$ as

$\left(10^{-7} \times \frac{10^{24}}{10^{10}}\right)^{-1} \times\left(5 \times 10^{2}\right) \times T \approx 5 \times 10^{-5} \times T$. Hence, in order to expect that 3)' and 4)' hold, it suffices to consider that $T \approx 5$ seconds.

Also, we see, by (7) and (5), that, for $K_{0}\left(\subseteq K_{N}\right)$ such that $1 \leq \#\left[K_{0}\right] \ll N$,

$$
\begin{aligned}
& \bar{m}_{T}\left(\left\{t \in[0, T]: X_{k}^{\omega_{0}}(t) \in \Xi_{k}\left(\in B_{\mathbb{R}^{6}}\right), k \in K_{0}\right\}\right) \\
& =\bar{m}_{T}\left(\left\{t \in[0, T]: \pi_{k}\left(\psi_{t}^{E}\left(\omega_{0}\right) \in \Xi_{k}\left(\in B_{\mathbb{R}^{6}}\right), k \in K_{0}\right\}\right)\right. \\
& =\bar{m}_{T}\left(\left\{t \in[0, T]: \psi_{t}^{E}\left(\omega_{0}\right) \in\left(\left(\pi_{k}\right)_{k \in K_{0}}\right)^{-1}\left(\begin{array}{c}
\left.\left.\left.\times \Xi_{k}\right)\right\}\right) \\
k \in K_{0}
\end{array}\right)\right)\right. \\
& \approx \bar{v}_{E}\left(\left(\left(\pi_{k}\right)_{k \in K_{0}}\right)^{-1}\left(\begin{array}{c}
\left.\left.\times \Xi_{k}\right)\right) \\
k \in K_{0}
\end{array}\right)\right. \\
& \equiv\left(\bar{v}_{E} \circ\left(\left(\pi_{k}\right)_{k \in K_{0}}\right)^{-1}\right)\left(\begin{array}{c}
\times \Xi_{k} \\
k \in K_{0}
\end{array}\right) .
\end{aligned}
$$

Particularly, putting $K_{0}=\{k\}$, we see:

$$
\begin{aligned}
& \bar{m}_{T}\left(\left\{t \in[0, T]: X_{k}^{\omega_{0}}(t) \in \Xi\right\}\right) \approx\left(\bar{v}_{E} \circ \pi_{k}^{-1}\right)(\Xi) \\
& \left(\forall \Xi \in B_{\mathbb{R}^{6}}\right) .
\end{aligned}
$$

Hence, we can describe the 3 ) and 4) in terms of $\left\{\pi_{n}\right\}_{n=1}^{N}$ in what follows.

Hypothesis $A[3)$ and 4)]. Put $K_{N}=\left\{1,2, \cdots, N\left(\approx 10^{24}\right)\right\}$. Let $H, E, v_{E}, \bar{v}_{E}, \pi_{k}: \Omega_{E} \rightarrow \mathbb{R}^{6}$ be as in the above. Then, summing up 3 ) and 4), by (9) we have:

(G) $\left\{\pi_{k}: \Omega_{E} \rightarrow \mathbb{R}^{6}\right\}_{k=1}^{N}$ is approximately independent random variables with the identical distribution in the 
sense that there exists $\rho_{E} \in M_{+1}^{m}\left(\mathbb{R}^{6}\right)$ such that

$$
\begin{aligned}
& \underset{k \in K_{0}}{\otimes} \rho_{E}(=\text { “product measure” }) \\
& \approx \bar{v}_{E} \circ\left(\left(\pi_{k}\right)_{k \in K_{0}}\right)^{-1} .
\end{aligned}
$$

for all $K_{0} \subset K_{N}$ and $1 \leqq \#\left[K_{0}\right] \ll N$.

Also, a state $(q, p)\left(\in \Omega_{E}\right)$ is called an equilibrium state if it satisfies $D_{K_{N}}^{(q, p)} \approx \rho_{E}$.

\subsubsection{Ergodic Hypothesis}

Now, we have the following theorem ( $c f .[2,3])$ :

Theorem A [Ergodic hypothesis]. Assume Hypothesis $A$ (or equivalently, 3$)$ and 4$)$ ). Then, for any $\omega_{0}=(q(0), p(0)) \in \Omega_{E}$, it holds that

$$
\begin{aligned}
& {\left[D_{K_{N}}^{(q(t), p(t))}\right](\Xi) \approx \bar{m}_{T}\left(\left\{t \in[0, T]: X_{k}^{\omega_{0}}(t) \in \Xi\right\}\right)} \\
& \left(\forall \Xi \in B_{\mathbb{R}^{6}}, k=1,2, \cdots, N\left(\approx 10^{24}\right)\right)
\end{aligned}
$$

for almost all $t$. That is,

$$
0 \leqq \bar{m}_{T}(\{t \in[0, T]:(12) \text { does not hold }\}) \ll 1 .
$$

Proof. Let $K_{0} \subset K_{N}$ such that $1 \ll \#\left[K_{0}\right] \equiv N_{0} \ll N$ (that is, $\frac{1}{\#\left[K_{0}\right]} \approx 0 \approx \frac{\#\left[K_{0}\right]}{N}$ ). Then, from Hypothesis A, the law of large numbers ( $c f .[12])$ says that

$$
D_{K_{0}}^{(q(t), p(t))} \approx \bar{v}_{E} \circ \pi_{k}^{-1}\left(\approx \rho_{E}\right)
$$

for almost all time $t$. Consider the decomposition $K_{N}=$ $\left\{K_{(1)}, K_{(2)}, \cdots, K_{(L)}\right\}$. (i.e., $K_{N}=\bigcup_{l=1}^{L} K_{(l)}$, $\left.K_{(l)} \cap K_{\left(l^{\prime}\right)}=\varnothing\left(l \neq l^{\prime}\right)\right)$, where $\#\left[K_{(l)}\right] \approx N_{0}$ $(l=1,2, \cdots, L)$. From (13), it holds that, for each $k$ $\left(=1,2, \cdots, N\left(\approx 10^{24}\right)\right)$,

$$
\begin{aligned}
& D_{K_{N}}^{(q(t), p(t))}=\frac{1}{N} \sum_{l=1}^{L}\left[\#\left[K_{(l)}\right] \times D_{K_{(l)}^{(t), p(t))}}^{(q(t)}\right. \\
& \approx \frac{1}{N} \sum_{l=1}^{L}\left[\#\left[K_{(l)}\right] \times \rho_{E}\right] \approx \bar{v}_{E} \circ \pi_{k}^{-1}\left(\approx \rho_{E}\right),
\end{aligned}
$$

for almost all time $t$. Thus, by (10), we get (12). Hence, the proof is completed.

We believe that Theorem A is just what should be represented by the "ergodic hypothesis" such that

$$
\begin{aligned}
& \text { "population average of } N \text { particles at each } t \text { " } \\
& =\text { "time average of one particle" }
\end{aligned}
$$

Thus, we can assert that the ergodic hypothesis is related to equilibrium statistical mechanics ( $c f$. the 2) in the abstract). Here, the ergodic property 2)' (or equivalently, equality (5)) and the above ergodic hypothesis should not be confused. Also, it should be noted that the ergodic hypothesis does not hold if the box (containing particles) is too large.
Remark 3 [The law of increasing entropy]. The entropy $H(q, p)$ of a state $(q, p)\left(\in \Omega_{E}\right)$ is defined by

$$
H(q, p)=k \log \left[v_{E}\left(\left\{\left(q^{\prime}, p^{\prime}\right) \in \Omega_{E}: D_{K_{N}}^{(q, p)} \approx D_{K_{N}}^{\left(q^{\prime}, p^{\prime}\right)}\right\}\right)\right]
$$

where

$$
k=[\text { Boltzmannconstant }] /\left([\text { Plank constant }]^{3 N} N !\right)
$$

Since almost every state in $\Omega_{E}$ is equilibrium, the entropy of almost every state is equal $k \log v_{E}\left(\Omega_{E}\right)$. Therefore, it is natural to assume that the law of increasing entropy holds.

\subsection{Statements Concerning Axiom 1 (Probabilistic Aspect; Measurement)}

In this section we shall study the probabilistic aspects of equilibrium statistical mechanics. For completeness, note that

(H) the argument in the previous section is not related to probability

since Axiom 1 does not appear in Section 3.1. Also, recall the $\left(\mathrm{E}_{4}\right)$, that is, there is no probability without measurement.

Note that the (12) implies that the equilibrium statistical mechanical system at almost all time $t$ can be regarded as:

(I) a box including about $10^{24}$ particles such as the number of the particles whose states belong to $\Xi\left(\in B_{\mathbb{R}^{6}}\right)$ is given by $\rho_{E}(\Xi) \times 10^{24}$.

Thus, it is natural to assume as follows.

(J) if we, at random, choose a particle from $10^{24}$ particles in the box at time $t$, then the probability that the state $\left(q_{1}, q_{2}, q_{3}, p_{1}, p_{2}, p_{3}\right)\left(\in \mathbb{R}^{6}\right)$ of the particle belongs to $\Xi\left(\in B_{\mathbb{R}^{6}}\right)$ is given by $\rho_{E}(\Xi)$.

In what follows, we shall represent this (J) in terms of measurements. Define the observable $\mathrm{O}_{0}=\left(\mathbb{R}^{6}, B_{\mathbb{R}^{6}}, F_{0}\right)$ in $C\left(\Omega_{E}\right)$ such that

$$
\left[F_{0}(\Xi)\right](q, p)=\left[D_{K_{N}}^{(q, p)}\right](\Xi)\left(\equiv \frac{\#\left[\left\{k \mid \pi_{k}(q, p) \in \Xi\right\}\right]}{\#\left[K_{N}\right]}\right)
$$

$\left(\forall \Xi \in B_{\mathbb{R}^{6}}, \forall(q, p) \in \Omega_{E}\left(\subset \mathbb{R}^{6 N}\right)\right)$.

Thus, we have the measurement

$\mathrm{M}_{C\left(\Omega_{E}\right)}\left(\mathrm{O}_{0}:=\left(\mathbb{R}^{6}, B_{\mathbb{R}^{6}}, F_{0}\right), S_{\left[\delta_{\psi_{t}}\left(q_{0}, p_{0}\right)\right]}\right)$. Then we say, by Axiom 1, that

$(\mathrm{K})$ the probability that the measured value obtained by the measurement

$\mathrm{M}_{C\left(\Omega_{E}\right)}\left(\mathrm{O}_{0}:=\left(\mathbb{R}^{6}, B_{\mathbb{R}^{6}}, F_{0}\right), S_{\left[\delta_{\psi_{t}}\left(q_{0}, p_{0}\right)\right]}\right)$ belongs to $\Xi\left(\in B_{\mathbb{R}^{6}}\right)$ is given by $\rho_{E}(\Xi)$. That is because Theorem A says that $\left[F_{0}(\Xi)\right]\left(\psi_{t}\left(q_{0}, p_{0}\right)\right) \approx \rho_{E}(\Xi)$ (almost every time $t$ ). 
Also, let $\Psi_{t}^{E}: C\left(\Omega_{E}\right) \rightarrow C\left(\Omega_{E}\right)$ be a deterministic Markov operator determined by the continuous map $\psi_{t}^{E}: \Omega_{E} \rightarrow \Omega_{E}$ (cf. Section 3.1.2). Then, it clearly holds $\Psi_{t}^{E} \mathrm{O}_{0}=\mathrm{O}_{0}$. And, we must take a $\mathrm{M}_{C\left(\Omega_{E}\right)}\left(\mathrm{O}_{0}, S_{\left[\left(q\left(t_{k}\right), p\left(t_{k}\right)\right)\right]}\right)$ for each time $t_{1}, t_{2}, \cdots, t_{k}, \cdots, t_{n}$. However, Interpretation $\left(E_{2}\right)$ says that it suffices to take the simultaneous measurement

$\mathrm{M}_{C\left(\Omega_{E}\right)}\left(\times_{k=1}^{n} \mathrm{O}_{0}, S_{\left[\delta_{(q(0), p(0))}\right]}\right)$. Here, for the simultaneous observable $\times_{k=1}^{n} \mathrm{O}_{0}$, see, for instance, Examples 1 and 3 in [4].

Remark 4. [The principle of equal a priori probabilities]. The (J) (or equivalently, $(\mathrm{K})$ ) says choose a particle from $N$ particles in box, and not choose a state from the state space $\Omega_{E}$. Thus, as mentioned in the abstract, the principle of equal (a priori) probability is not related to our method. If we try to describe Ruele's method [7] in terms of measurement theory, we must use statistical measurement theory $(c f .[2,6])$. However, this trial will end in failure. Also, our recent report [15] will promote the understanding of measurement theory.

\section{Conclusions}

Our concern in this paper may be regarded as the problem: "What is the classical mechanical world view?" Concretely speaking, we are concerned with the problem: " $\left(\mathrm{B}_{2}\right)$ vs. $\left(\mathrm{B}_{3}\right)$ ", and thus, "our method $[2,3]$ vs. Ruele's method [7]". In this paper, we added important remarks (i.e., Remarks 1 and 2) to our method [2,3], and strengthened our method in the light of the mechanical world view $[4,5]$.

Equilibrium statistical mechanics is of course one of the most fundamental theories in science. And it is sure that Ruele's method [7] has been authorized for a long time. Therefore, we hope that our proposal will be examined from various view points.

\section{REFERENCES}

[1] S. Ishikawa, "A Quantum Mechanical Approach to a
Fuzzy Theory," Fuzzy Sets and Systems, Vol. 90, No. 3 , 1997, pp. 277-306. doi:10.1016/S0165-0114(96)00114-5

[2] S. Ishikawa, "Mathematical Foundations of Measurement Theory," Keio University Press Inc., Tokyo, 2006.

[3] S. Ishikawa, "Ergodic Problem in Quantitative Language," Far East Journal of Dynamical Systems, Vol. 11, No. 1, 2009, pp. 33-48.

[4] S. Ishikawa, "A New Interpretation of Quantum Mechanics," Journal of Quantum Information Science, Vol. 1, No. 2, 2011, pp. 35-42.

[5] S. Ishikawa, "Quantum Machanics and the Philosophy of Language: Reconsideration of Traditional Philosophies," Journal of Quantum Information Science, Vol. 2, No. 1, 2012, pp. 2-9.

[6] S. Ishikawa, "A Measurement Theoretical Foundation of Statistics," Journal of Applied Mathematics, Vol. 3, No. 3, 2012, pp. 283-292.

[7] D. Ruelle, "Statistical Mechanics, Rigorous Results," World Scientific, Singapore, 1969.

[8] G. Gallavotti, "Statistical Mechanics: A Short Treatise," Springer Verlag, Berlin, 1999.

[9] M. Toda, R. Kubo and N. Saito, "Statistical physics, Springer Series in Solid-State Sciences," Springer Verlag, Berlin, 1983.

[10] G. J. Murphy, "C$C^{*}$-Algebras and Operator Theory," Academic Press, Waltham, 1990.

[11] J. von Neumann, "Mathematical Foundations of Quantum Mechanics," Springer Verlag, Berlin, 1932.

[12] A. N. Kolmogorov, "Foundations of the Theory of Probability (Translation)," 2nd Edition, Chelsea Pub Co, New York, 1960.

[13] E. B. Davies, "Quantum Theory of Open Systems," Academic Press, Waltham, 1976.

[14] U. Krengel, "Ergodic Theorems," Walter de Gruyter, Berlin, 1985. doi:10.1515/9783110844641

[15] S. Ishikawa, "The Linguistic Interpretation of Quantum Mechanics," 2012. http://arxiv.org/abs/1204.3892 